\title{
Uma proposta para avaliação da sustentabilidade socioambiental utilizando suporte analítico e gráfico
}

\author{
Camila Duarte Teles ${ }^{\mathrm{a} *}$, Camila Costa Dutra ${ }^{\mathrm{a}}$, José Luis Duarte Ribeiro ${ }^{\mathrm{a}}$, \\ Lia Buarque de Macedo Guimarães ${ }^{a}$ \\ anniversidade Federal do Rio Grande do Sul, Porto Alegre, RS, Brasil \\ *camila.teles@bento.ifrs.edu.br
}

\begin{abstract}
Resumo
Este trabalho apresenta um procedimento para auxiliar as empresas na avaliação da sustentabilidade socioambiental, aspecto que tem se mostrado essencial para a competitividade das mesmas. 0 procedimento desenvolvido emprega suporte analítico e gráfico para avaliação das dimensões ambiental e social das empresas, considerando tanto o desempenho interno quanto o externo. 0 desenvolvimento do procedimento contemplou cinco etapas: identificação dos itens de avaliação; organização dos itens em dimensões a serem analisadas; aplicação de questionário; totalização e visualização gráfica dos resultados. 0 questionário foi aplicado em 12 empresas. Para a visualização gráfica dos resultados, foi utilizado um gráfico radar, no qual os eixos representam as dimensões social, ambiental, interna e externa. As empresas pesquisadas revelaram melhor desempenho socioambiental externo, provavelmente em virtude da preocupação com a sua imagem. Além disso, verificou-se que empresas exportadoras possuem melhor desempenho socioambiental, fato que pode ser oriundo da maior concorrência e exigência do mercado externo.
\end{abstract}

Palavras-chave

Avaliação da sustentabilidade. Sustentabilidade socioambiental. Responsabilidade social. Gestão ambiental.

\section{Introdução}

Apesar de a importância dada ao desenvolvimento sustentável ter aumentado nos últimos anos, ainda há uma série de desafios associados a esse assunto, tanto nos países desenvolvidos quanto nos países em desenvolvimento e recém-industrializados. Mesmo com os esforços de instituições internacionais e governamentais, tem sido difícil transformar o conceito de desenvolvimento sustentável em realidade (Veiga \& Magrini, 2009).

A sustentabilidade é alcançada através da promoção do desenvolvimento sustentável, sendo que este último pode ser promovido a partir de uma ampla variedade de disciplinas, todas elas necessárias para atingir o objetivo final (Garcia-Serna et al., 2007). Para Hart \& Milstein (2004), uma empresa sustentável é aquela que gera, simultaneamente, benefícios econômicos, sociais e ambientais, conhecidos como os três pilares da sustentabilidade, contribuindo com o desenvolvimento sustentável.
Além das ferramentas usuais que auxiliam no alcance da sustentabilidade empresarial, verifica-se a necessidade de formas de avaliação da sustentabilidade, para permitir a análise da situação econômica, ambiental e social das empresas. Böhringer \& Jochem (2007) corroboram essas afirmações, dizendo que atualmente há um forte interesse na avaliação da prosperidade econômica, da qualidade ambiental e da coerência social.

A demanda por formas de avaliação da sustentabilidade foi identificada na Rio92, que enfatizou a necessidade do desenvolvimento de indicadores de sustentabilidade para auxiliar os países, a comunidade e as empresas na tomada de decisão relacionada ao Desenvolvimento Sustentável. Isso, porque, embora o conceito de sustentabilidade seja compreendido intuitivamente, é difícil expressá-lo em nível operacional (Labuschagne et al., 2005). Apesar do tempo transcorrido desde a identificação 
desta necessidade, Ugwu \& Haupt (2007) afirmam que ainda há uma demanda intensa por formas de avaliação da sustentabilidade.

Além disso, como as Nações Unidas e os governos dos países foram a força motriz do desenvolvimento sustentável, a maior parte das formas de avaliação da sustentabilidade desenvolvidas é direcionada para os níveis nacional, regional ou de comunidade, sendo que um número menor de trabalhos foram realizados em nível empresarial. Destes, a maioria tem foco no produto, sem fornecer indicação da sustentabilidade da empresa como um todo. Além disso, abordam principalmente a dimensão ambiental, referindo-se parcialmente às dimensões econômica e social (Labuschagne et al., 2005).

Os dados de uma pesquisa sobre gestão ambiental, realizada com 412 das mil maiores empresas do Brasil, indicam que as mesmas estão preocupadas com as questões ambientais, existindo intenso movimento no meio empresarial na busca da ecoeficiência e, consequentemente, da sustentabilidade ambiental. Entretanto, as empresas também necessitam ser economicamente sustentáveis, visto que seu maior compromisso é gerar lucro, e socialmente sustentáveis, trazendo retorno para a sociedade. Apesar desta tendência e da evolução das empresas do ponto de vista ambiental, as mesmas ainda não estão preparadas ou organizadas para alcançar a sustentabilidade, havendo muitas atividades a serem feitas (Análise Editorial, 2007). Assim, observa-se a demanda pelo desenvolvimento de instrumentos que auxiliem as empresas na busca da sustentabilidade, tais como as formas de avaliação.

Segundo Böhringer \& Jochem (2007), o que não pode ser claramente medido é difícil melhorar ou atingir. Assim, a avaliação da sustentabilidade representa um papel fundamental no alcance da sustentabilidade, visto que indicam os pontos fracos e fortes das empresas em relação ao seu desempenho econômico, social e ambiental. A partir das informações de desempenho obtidas, as empresas podem tomar decisões direcionadas à sustentabilidade.

Considerando-se que a sustentabilidade consolida-se como um fator competitivo para as empresas, o objetivo deste trabalho é desenvolver um procedimento analítico e gráfico para as empresas avaliarem sua sustentabilidade. As dimensões da sustentabilidade a serem avaliadas são a ambiental e a social, sendo ambas desdobradas em aspectos internos e externos das empresas. Este trabalho está organizado em cinco seções. Após está introdução, as seções 2 e 3 discorrem sobre desenvolvimento sustentável e avaliação da sustentabilidade. A seção 4 apresenta o procedimento de avaliação da sustentabilidade desenvolvido e proposto neste artigo. A seção 5 relata e discute os resultados da aplicação prática do procedimento proposto, enquanto a seção 6 sumariza as conclusões do trabalho.

\section{Desenvolvimento sustentável}

0 conceito de desenvolvimento foi redefinido pela World Commission on Environment and Development (1987, p. 41) Mundial sobre Meio Ambiente e Desenvolvimento da Organização das Nações Unidas em 1987, no relatório Nosso Futuro Comum. Segundo este documento, desenvolvimento sustentável é "aquele capaz de atender às necessidades das atuais gerações sem comprometer os direitos das futuras gerações".

A Agenda 21, documento (Brasil, 1992) elaborado na Conferência das Nações Unidas sobre o Meio Ambiente (também conhecida como Rio92), traduz em ações o desenvolvimento sustentável, concentra-se nas dimensões ambiental, social e econômica da sustentabilidade. Logo, para uma sociedade ser dita sustentável, deve atender aos seguintes critérios: ser viável economicamente, gerando desenvolvimento econômico de forma igualitária; produzir sem agredir o meio ambiente, ocorrendo preservação e conservação dos recursos naturais existentes; contribuir para 0 desenvolvimento social, gerando qualidade de vida, bem-estar social e educação, resultando em uma sociedade saudável (Cantarino et al., 2007; Programa das Nações Unidas para o Meio Ambiente, 2002). Embora haja conflito sobre seu significado exato, muitos autores concordam que o desenvolvimento sustentável refere-se realmente à satisfação das dimensões sociais, ambientais e econômicas (Azapagic \& Perdan, 2000).

No âmbito empresarial, assim como ocorre na sociedade em geral, para alcançar o desenvolvimento sustentável corporativo, as empresas necessitam ser sustentáveis ambiental, social e economicamente. A verdadeira sustentabilidade corporativa exige a integração dessas três dimensões na gestão das empresas, o que pode levar a transformações no modelo empresarial para assegurar a sustentabilidade das operações em longo prazo. Verifica-se que o desenvolvimento sustentável demanda das empresas, entre outros elementos: capacitação dos empregados e da comunidade na qual está inserida; participação em projetos comunitários e ambientais na região em que está instalada; adoção de estratégias empresariais que assegurem o lucro do empreendimento hoje, protegendo, sustentando e aumentando os recursos humanos e naturais que serão necessários no futuro, e agregação de valor na economia do país, no qual a empresa está realizando seus negócios (Labuschagne et al., 2005; Brasil, 2007; Weber, 2008). 
As principais razões para integrar a sustentabilidade na prática empresarial são: (i) redução de custos proveniente da utilização de métodos de produção mais limpa e inovação, que melhoram a eficiência dos recursos; (ii) redução de custos relacionados a saúde e segurança, visto que melhorias no bem-estar dos trabalhadores e da comunidade aumentam a produtividade e reduzem custos com serviços sociais; (iii) redução nos custos com mão de obra, já que boas condições de trabalho melhoram a motivação e a produtividade, e diminuem o absentismo e a rotação de empregados; (iv) fácil acesso a instituições de crédito, uma vez que riscos menores levam a taxas de empréstimo e custos de seguros mais baixos; (v) reputação da empresa, já que o compromisso com o desenvolvimento sustentável melhora sua reputação; (vi) vantagem no mercado, uma vez que a gestão integrada da cadeia de suprimentos permite o desenvolvimento de relações mais profundas com os clientes; (vii) atração de investidores éticos, que excluem as empresas associadas a um desempenho social e ambiental inaceitável (Azapagic, 2003; Phillis \& Davis, 2009).

Embora o conceito de desenvolvimento sustentável seja relativamente fácil de compreender, as dificuldades aparecem na tentativa de aplicar os princípios do mesmo na prática. Uma das dificuldades é a necessidade de medir o nível de sustentabilidade dos diferentes setores da sociedade, ou seja, dos governos locais e nacionais, da indústria, das comunidades locais e dos indivíduos, para determinar quais mudanças conduzem à sustentabilidade. Assim, como endossado no Capítulo 40 da Agenda 21, é necessário desenvolver indicadores adequados de desenvolvimento sustentável que permitam essa avaliação. Até o momento, diferentes abordagens foram propostas para definir indicadores para diferentes partes da comunidade, incluindo a indústria. No entanto, ainda não existe uma metodologia padronizada com um conjunto de indicadores genéricos que permita uma comparação consistente e a identificação das opções mais sustentáveis (Azapagic \& Perdan, 2000).

\section{Avaliação da sustentabilidade}

Reconhecendo-se que não se pode gerenciar aquilo que não é medido, é importante medir o desempenho de um determinado sistema para verificar se o mesmo está alcançando suas metas e compará-lo com outros. Essa avaliação fornece as informações necessárias para a tomada de decisões, o alcance de um padrão de referência e a promoção da melhoria contínua (Veleva et al., 2001).

A finalidade da avaliação da sustentabilidade é fornecer aos tomadores de decisão uma avaliação dos sistemas em termos de perspectivas de curto e longo prazo, a fim de ajudá-los a determinar quais ações devem ou não devem ser realizadas na tentativa de tornar a sociedade sustentável. Além disso, a avaliação da sustentabilidade tem o propósito de comunicar aos stakeholders os esforços e o progresso de uma organização nas dimensões econômica, ambiental e social (Böhringer \& Jochem, 2007; Lozano, 2006; Ness et al., 2007; Singh et al., 2009; Wilson et al., 2007).

Os indicadores de sustentabilidade permitem a identificação de opções mais sustentáveis através de: i) comparação de produtos semelhantes feitos por diferentes empresas; ii) comparação de diferentes processos que produzem o mesmo produto; iii) benchmarking das unidades de uma corporação; iv) avaliação de uma empresa frente a outras empresas do mesmo setor; v) avaliação do progresso em direção ao desenvolvimento sustentável de um setor (Krajnc \& Glavic, 2003).

As medidas de avaliação da sustentabilidade de macronível ou nível econômico cobrem as abordagens e os métodos que adaptam as estratégias convencionais dos tomadores de decisão regionais, nacionais ou internacionais para aquelas sustentáveis. Estes tomadores de decisão podem ser governos, suas respectivas unidades ou organizações responsáveis pelo desenvolvimento econômico e pela proteção do ambiente. Várias ferramentas têm sido propostas para avaliar a sustentabilidade neste nível, como o framework do CDS das Nações Unidas e os indicadores da Agência de Meio ambiente Europeia (Wang \& Lin, 2004).

No nível empresarial, tradicionalmente, os indicadores financeiros e de qualidade (por exemplos, valor presente líquido - VPL; taxa interna de retorno - TIR; análise custo-benefício; fatia de mercado; taxa de retorno; taxa de defeito nos produtos) têm sido utilizados para avaliar o desempenho. Entretanto, com o crescimento do movimento da sustentabilidade, as empresas estão percebendo a importância de medir e melhorar seu desempenho ambiental e social. lsso pode economizar recursos e melhorar a imagem da empresa, gerar vantagem competitiva e, assim, aumentar os lucros (Veleva et al., 2001; Wang \& Lin, 2004; Herzig \& Schaltegger, 2006).

Um número crescente de estudos revela a relação entre um bom desempenho socioambiental e os resultados financeiros. Entre os mais importantes objetivos e benefícios de avaliar e reportar a sustentabilidade, estão: legitimação das atividades, dos produtos e dos serviços corporativos, que geram impactos ambientais e sociais; aumento da reputação corporativa e do valor da marca; ganho de vantagem competitiva; comparação e benchmarking com concorrentes; aumento da 
transparência e responsabilidade dentro da empresa; motivação dos funcionários. Além disso, com a ajuda da Internet, os clientes hoje estão mais bem informados e precisam ser constantemente convencidos de que uma empresa está agindo corretamente (Veleva et al., 2001; Wang \& Lin, 2004; Herzig \& Schaltegger, 2006). Nos últimos anos, tem havido uma crescente pressão dos stakeholders para as empresas se tornarem mais transparentes e divulgarem informações sobre seu desempenho relacionado à sustentabilidade (Darby \& Jenkins, 2006).

Em função disso, um número crescente de empresas está comprometendo-se com a responsabilidade socioambiental e, logo, estas necessitam de novas ferramentas para avaliar seu progresso. Este crescente interesse da indústria na sustentabilidade resultou no desenvolvimento de ferramentas que, atualmente, colocam ênfase apenas no ambiente e, portanto, são mais indicadores de desempenho ambiental do que de desenvolvimento sustentável (Azapagic \& Perdan, 2000). As principais ferramentas de avaliação ambiental são: análise de custo-benefício; avaliação ambiental; avaliação de riscos, e análise do ciclo de vida (ACV). Entre estas, a ACV é talvez a abordagem mais amplamente aceita, mas o alcance limitado e as funções da ACV para medições ambientais reduzem a força e a abrangência do método. Com base nessas ferramentas, várias métricas ou critérios estão sendo desenvolvidos por diversas empresas (Veleva et al., 2001; Wang \& Lin, 2004).

Embora tenha havido numerosas tentativas de desenvolver indicadores de sustentabilidade empresariais, para avaliar as contribuições corporativas para a sustentabilidade, não se alcançou consenso sobre um conjunto comum de indicadores. Além disso, muitas dessas abordagens são difíceis de aplicar ou têm significado limitado. 0 Global Reporting Initiative (GRI), o mais conhecido framework para relatório voluntário de desempenho ambiental e social por empresas e outras organizações em nível mundial, tem atualmente o melhor potencial para se tornar uma ferramenta dessa natureza; no entanto, não fornece uma visão ou orientação clara para a aplicação prática. Além disso, seu framework é complexo e os requisitos do relatório são extremamente onerosos para as empresas de pequeno e médio porte (Figge \& Hahn, 2004; Azapagic \& Perdan, 2000; Krajnc \& Glavic, 2005; Azapagic \& Perdan, 2000; Brown et al., 2009).

Verifica-se, assim, a necessidade de se desenvolver um framework para avaliação da sustentabilidade das empresas. No entanto, dado o número de indicadores que devem ser considerados e o número de tomadores de decisão ou stakeholders que podem potencialmente ser envolvidos no processo de tomada de decisão, o problema de identificar as melhores opções em uma determinada situação não é trivial. Em função disso, o número de ferramentas para avaliação da sustentabilidade aumentou nos últimos anos (Veleva et al., 2001; Azapagic \& Perdan, 2000; Brown et al., 2009; Böhringer \& Jochem, 2007; Wilson et al., 2007).

Em seus trabalhos, Gasparatos et al. (2008), Ness et al. (2007), Singh et al. (2009), Parris \& Kates (2003), Krajnc \& Glavic (2005) e Labuschagne et al. (2005) citam diversas ferramentas de avaliação da sustentabilidade, tais como: indicadores da Comissão das Nações Unidas para o Desenvolvimento Sustentável (CNUDS); Índice de Sustentabilidade Ambiental; Índice de Bem-Estar (Wellbeing Index); Pegada Ecológica; Indicador de Progresso Genuíno; Análise de Emergia; Análise de Exergia; Índice de Bem-Estar Econômico Sustentável; Poupança Genuína; Índice de Desenvolvimento Humano; Produto Nacional Líquido Verde (Green Net National Product); Índice de Desempenho de Sustentabilidade; Índice de Planeta Vivo; Índice de Amizade Ambiental; Índice de Sustentabilidade Ambiental. Já para avaliação da sustentabilidade empresarial, as ferramentas mais citadas pelos mesmos autores são Global Reporting Initiative (GRI), Índice de Sustentabilidade Dow Jones, Institute of Chemical Engineers (IChemE) Sustainability Metrics e Wuppertal Sustainable Development Indicator Framework, Índice de Sustentabilidade Empresarial da Bovespa e Indicadores Ethos de Responsabilidade Social Empresarial. A Tabela 1 apresenta as principais lacunas dessas ferramentas.

Sumarizando, as principais deficiências dessas ferramentas de avaliação da sustentabilidade empresarial são: focar uma dimensão em detrimento das demais; ser extensa; não sumarizar um índice; avaliar o desempenho de determinado grupo de empresas; ser orientado ao desempenho financeiro das empresas. Além disso, não são ferramentas visuais que permitem a interpretação dos resultados facilmente e não fragmentam as dimensões econômica, ambiental e social em interna e externa, não orientando as ações para melhoria do desempenho das empresas.

Foram encontradas na literatura duas ferramentas visuais para avaliação da sustentabilidade, que são o Dashboard of Sustainability (Hardi \& Zdan, 2000) e o Barometer of Sustainability (Prescott-Allen, 1997). Os autores do Dashboard of Sustainability ressaltam: para que a ferramenta se torne mais relevante e atrativa o suficiente para envolvidos no processo de avaliação de sustentabilidade, os indicadores utilizados precisam ser substituídos por um grupo de indicadores reconhecidos internacionalmente. Já o Barometer of Sustainabilityé criticado por muitos autores por não ser um método científico (Bossel, 1999). 
Tabela 1. Lacunas das ferramentas de avaliação da sustentabilidade empresarial.

\begin{tabular}{|c|c|}
\hline $\begin{array}{l}\text { Ferramenta de avaliação da } \\
\text { sustentabilidade }\end{array}$ & Lacunas \\
\hline Global Reporting Initiative (GRI) & $\begin{array}{l}\text { Concentra-se em apresentar diretrizes para elaboração de um relatório de sustentabilidade } \\
\text { Singh et al. (2009). } \\
\text { Apresenta mais de } 100 \text { indicadores, sendo que nem todos são fáceis de avaliar Labuschagne et al. } \\
\text { (2005). } \\
\text { A empresa indica o nível de aplicação das diretrizes Instituto Ethos de Empresas e Responsabilidade } \\
\text { Social, (2006a). } \\
\text { Não apresenta um índice Instituto Ethos de Empresas e Responsabilidade Social (2006a). }\end{array}$ \\
\hline $\begin{array}{l}\text { Indicadores Ethos de Responsabilidade } \\
\text { Social Corporativa }\end{array}$ & $\begin{array}{l}\text { Questionário extenso, } 40 \text { indicadores desdobrados em mais de } 400 \text { perguntas Instituto Ethos de } \\
\text { Empresas e Responsabilidade Social (2006b). } \\
\text { Não apresenta um índice Instituto Ethos de Empresas e Responsabilidade Social 2006b). }\end{array}$ \\
\hline $\begin{array}{l}\text { Institute of Chemical Engineers (1ChemE) } \\
\text { Sustainability Metrics }\end{array}$ & $\begin{array}{l}\text { Concentra-se nos aspectos ambientais e em indicadores quantificáveis que podem não ser praticáveis } \\
\text { em todas as empresas Labuschagne et al. (2005). }\end{array}$ \\
\hline $\begin{array}{l}\text { Wuppertal Sustainable Development } \\
\text { Indicator Framework }\end{array}$ & $\begin{array}{l}\text { Poucos indicadores para avaliação da dimensão social da sustentabilidade empresarial } \\
\text { Labuschagne et al. (2005). }\end{array}$ \\
\hline Índice de Sustentabilidade Dow Jones & $\begin{array}{l}\text { Questionário extenso, contendo mais de } 70 \text { perguntas } \\
\text { Claramente composto de itens que podem afetar o sucesso financeiro da empresa } \\
\text { Tem como objetivo acompanhar a performance financeira das empresas líderes em termos de } \\
\text { sustentabilidade empresarial } \\
\text { Poucas questões relacionadas à dimensão ambiental } \\
\text { Poucos critérios relacionados à dimensão social associados à sociedade Labuschagne et al. (2005). }\end{array}$ \\
\hline $\begin{array}{l}\text { Índice de Sustentabilidade Empresarial } \\
\text { da Bovespa }\end{array}$ & $\begin{array}{l}\text { Questionário extenso, } 61 \text { indicadores desdobrados em várias perguntas BM\&FBOVESPA (2008). } \\
\text { Tem por objetivo refletir o retorno de uma carteira composta por ações de empresas com reconhecido } \\
\text { comprometimento com a responsabilidade social e a sustentabilidade empresarial BM\&FBOVESPA } \\
\text { (2008). } \\
\text { Avalia o desempenho das companhias emissoras das } 150 \text { ações mais negociadas da BOVESPA } \\
\text { BM\&FBOVESPA (2008). }\end{array}$ \\
\hline
\end{tabular}

Fonte: elaborado pelos autores.

\section{Proposta de procedimento analítico e gráfico para avaliação socioambiental}

0 desenvolvimento do procedimento analítico e gráfico para a avaliação socioambiental contemplou cinco etapas, sendo a primeira a identificação dos itens de avaliação. Posteriormente, realizou-se a organização dos itens em dimensões a serem analisadas, seguida da aplicação do questionário e da totalização dos resultados. Por fim, foi realizada a visualização gráfica dos resultados. Estas etapas são descritas a seguir.

\subsection{Identificação dos critérios e itens de avaliação}

A identificação dos critérios socioambientais e seus respectivos itens de avaliação baseou-se nos Indicadores Ethos de Responsabilidade Social Corporativa, no questionário do ISE (Índice de Sustentabilidade Empresarial da Bovespa) e no questionário da Revista Amanhã, (Instituto Ethos de Empresas e Responsabilidade Social, 2006b), (BM\&FBOVESPA, 2008), (Revista Amanhã, 2008) os quais foram desenvolvidos para avaliação da sustentabilidade socioambiental. Os critérios abordados por estes questionários são: valores, transparência e governança; público interno; meio ambiente; fornecedores; consumidores e clientes; comunidade; governo e sociedade. 0 questionário da Revista Análise - Gestão Ambiental - Anuário 2007 (Análise Editorial, 2007) também foi utilizado para embasamento das questões ambientais. Contudo, estes questionários são extensos e complexos, o que pode provocar a desmotivação dos respondentes. Considerando-se esses fatores, o presente estudo procurou sumarizar os principais itens de sustentabilidade socioambiental contidos nestes questionários.

É importante ressaltar que a lista resume os princípios de sustentabilidade socioambiental mais importantes, sendo uma ferramenta pró-ativa para a gestão socioambiental e, no seu conjunto, uma ferramenta para a comparação entre diferentes empresas. 0 estudo não envolveu a dimensão econômica, uma vez que as empresas e a literatura já possuem meios consolidados para avaliá-la, tais como o valor presente líquido, a taxa interna de retorno ou o acompanhamento da fatia de mercado.

\subsection{Organização dos itens em dimensões a serem analisadas}

De acordo com esta proposta, os itens identificados foram organizados em quatro quadrantes: social interno (SI) e social externo (SE), que correspondem às práticas sociais adotadas nos ambientes interno e externo da empresa, respectivamente; ambiental 
interno (Al) e ambiental externo (AE), que correspondem às práticas ambientais adotadas internamente $\mathrm{e}$ externamente à empresa. 0 Apêndice $A$ apresenta os 42 itens identificados e seus respectivos quadrantes.

\subsection{Aplicação do questionário}

A lista de itens foi adaptada para tornar-se um instrumento de avaliação a ser respondido pelas empresas quanto ao nível de sustentabilidade, ou seja, quais as práticas de gestão socioambiental são adotadas pelas empresas. 0 questionário elaborado contém 42 questões, às quais são atribuídas pontuação de 0 (zero) a 10 (dez), conforme a resposta da empresa participante do estudo.

As 42 questões são de três tipos distintos. 0 primeiro tipo de questão verifica a existência e a observância de uma prática, medida na escala 0 a 10; por exemplo, questão 5, que versa sobre a inclusão dos princípios do desenvolvimento sustentável no planejamento estratégico. 0 participante poderia responder essa questão utilizando a escala de 0 a 10, em que zero corresponde a "discordo plenamente" e dez a "concordo plenamente". As questões de 1 a 7 , de 13 a 16 , de 19 a 23 e de 25 a 42 enquadram-se neste tipo.

0 segundo tipo de questão verifica a abrangência da ação. Quanto maior o número de alternativas contempladas pela empresa, maior a pontuação na escala 0 a 10. Por exemplo, questão 9a, que verifica a abrangência da comunicação das ações sociais, contendo as alternativas: Não há programa; Escola; Público em geral; Vizinhança; ONGs; Autoridades e Outros; nesta questão, quem respondesse "não há programa de comunicação social” receberia pontuação zero, enquanto quem indicasse $x$ alternativas entre as $n$ possíveis receberia pontuação $10 x / n$. As questões 8 , 9a, 9b, 17, 18 e 24 enquadram-se neste tipo.

0 terceiro tipo de questão verifica o tempo de experiência ou maturidade referente a alguma prática; por exemplo, questão 11, que pergunta se a empresa possui $1 S 014.001$ ou norma equivalente (Associação Brasileira de Normas Técnicas, 2004), contendo as alternativas de resposta: não; não, mas planeja implementar; há 2 anos; de 2 a 5 anos; de 5 a 10 anos; há mais de 10 anos. Nesta questão, a resposta "não" recebe zero ponto; a resposta "não, mas pretende implementar" recebe 2 pontos; a resposta "há 2 anos" recebe 4 pontos, e assim por diante, até 10 pontos, para as empresas que possuem a norma ISO 14.001 ou equivalente há mais de 10 anos. As questões 10, 11 e 12 enquadram-se neste tipo.

0 foco principal da pesquisa foram as empresas de médio e grande porte, que deveriam possuir iniciativas voltadas para a sustentabilidade. Para permitir um amplo teste da proposta descrita neste artigo, definiu-se que a amostra deveria contemplar empresas médias e grandes de diversos setores industriais. Assim, foram selecionados os seguintes setores: Petroquímico, Alimentos, Automotivo, Eletroeletrônico, Máquinas e Implementos Agrícolas, Metalúrgico, Moveleiro, Serviços, Siderúrgico e Transporte. A versão final do questionário foi enviada por e-mail com opção de resposta na versão eletrônica para um grande número de empresas dos setores indicados. A coleta de dados foi encerrada após o retorno de ao menos um questionário de cada um dos setores listados. Essa conduta assegurou a diversidade de empresas desejada para o teste da proposta apresentada neste artigo.

\subsection{Procedimento analítico}

Para a totalização dos resultados, todas as questões receberam o mesmo peso unitário. 0 peso unitário de cada questão foi distribuído entre os quadrantes avaliados na mesma. Por exemplo, se uma questão abrange quatro quadrantes (social interno, social externo, ambiental interno e ambiental externo), o peso unitário é dividido em quatro. Assim, cada um dos quadrantes dessa questão recebe peso parcial de 0,25 . Já uma questão que abrange somente dois quadrantes, o seu peso unitário é dividido por dois, resultando, para cada um desses dois quadrantes, um peso parcial de 0,5 .

Uma vez definidos os pesos dos quadrantes em cada uma das questões, foi calculada a média ponderada das notas atribuídas pelas empresas às diversas questões. Os resultados obtidos foram empregados para a análise e a visualização dos dados. 0 eixo principal vertical, que representa as dimensões ambiental (A) e social (S), teve suas notas calculadas a partir da média simples dos quadrantes ambiental interno e externo, e social interno e externo, respectivamente. 0 eixo principal horizontal, que indica o desempenho socioambiental interno (DI) e externo (DE) da empresa, teve suas notas obtidas através da média simples dos quadrantes social e ambiental interno e social e ambiental externo, respectivamente.

\subsection{Procedimento gráfico}

Para a visualização gráfica dos resultados, foi utilizado um gráfico radar. Neste tipo de gráfico, os eixos de valores partem de um centro comum. Para este estudo, conforme explicado, o eixo principal vertical representa as dimensões social e ambiental, e o eixo principal horizontal representa o desempenho interno e externo. Os quadrantes (social interno, social 
externo, ambiental interno, ambiental externo) foram representados nos eixos secundários, desenhados a 45 graus a partir dos eixos principais. Uma linha faz a conexão das notas obtidas em cada avaliação, formando um polígono preenchido. A avaliação da sustentabilidade pode ser facilmente apreendida observando-se a área do polígono de oito lados. Quando maior a área do polígono, melhor é o desempenho de sustentabilidade da empresa. A forma do polígono também é relevante, uma vez que as assimetrias indicam que a empresa está obtendo melhor desempenho em relação a algum dos eixos, em detrimento dos demais.

\section{Resultados e discussão}

0 percentual de respostas obtidas das empresas foi de 16\% durante o período de dezembro de 2008 a janeiro de 2009, que corresponde a 12 empresas. Estas respostas encontram-se compiladas no Apêndice B. As empresas onde foi aplicado o procedimento foram caracterizadas segundo seu ramo de atividade, porte (em relação ao número de funcionários) e principais clientes, conforme apresentado na Tabela 2.

Na totalização dos resultados, obteve-se uma nota para cada um dos quadrantes (social interno - Sl, social externo - SE, ambiental interno - $\mathrm{Al}$, ambiental externo - AE). Também foram calculadas as notas das dimensões ambiental (A) e social (S) e do desempenho socioambiental interno (DI) e externo (DE). Estes resultados podem ser observados na Tabela 3.

Para a visualização gráfica dos resultados foram gerados gráficos radar para cada uma das empresas respondentes. Estes gráficos são apresentados na Figura 1, na ordem decrescente de desempenho de sustentabilidade.

As duas empresas com melhor desempenho socioambiental pertencem ao setor petroquímico e automotivo, setores que se destacam na economia brasileira em relação ao seu nível de desenvolvimento. Estas empresas possuem um equilíbrio entre as ações sociais e ambientais, tanto no ambiente interno quanto externo a empresa.

Tabela 2. Caracterização das empresas.

\begin{tabular}{ccll}
\hline Empresa & Ramo de atividade & Porte & Principais Clientes \\
\hline 1 & Petroquímico & Grande & Mercado interno, América e Europa \\
2 & Automotivo & Grande & Mercado interno, América, Europa, Ásia e Oceania \\
3 & Moveleiro & Grande & Mercado interno, América, Europa, Ásia, África e Oceania \\
4 & Máq. e lmpl. Agrícolas & Grande & Mercado interno e América \\
5 & Transporte & Médio & Mercado interno \\
6 & Metalúrgico & Grande & Mercado interno, América e África \\
7 & Alimentos & Grande & Mercado interno \\
8 & Eletroeletrônico & Grande & Mercado interno, América e Europa \\
9 & Metalúrgico & Médio & Mercado interno e América \\
10 & Serviços & Médio & Mercado interno \\
11 & Transporte & Médio & Mercado interno \\
12 & Siderúrgico & Grande & Mercado interno, América, Europa e Ásia \\
\hline
\end{tabular}

Fonte: elaborado pelos autores.

Tabela 3. Totalização dos resultados.

\begin{tabular}{|c|c|c|c|c|c|c|c|c|}
\hline Empresa & $\mathrm{SI}$ & SE & $\mathrm{Al}$ & $\mathrm{AE}$ & A & $\mathrm{S}$ & DI & $\mathrm{DE}$ \\
\hline 1 & 89 & 100 & 98 & 100 & 99 & 94 & 93 & 100 \\
\hline 2 & 94 & 95 & 96 & 95 & 95 & 94 & 95 & 95 \\
\hline 3 & 68 & 80 & 70 & 74 & 72 & 74 & 69 & 77 \\
\hline 4 & 78 & 83 & 72 & 77 & 75 & 80 & 75 & 80 \\
\hline 5 & 83 & 95 & 90 & 89 & 89 & 89 & 87 & 92 \\
\hline 6 & 43 & 39 & 52 & 41 & 47 & 41 & 47 & 40 \\
\hline 7 & 57 & 62 & 64 & 49 & 57 & 59 & 60 & 56 \\
\hline 8 & 88 & 90 & 90 & 81 & 86 & 89 & 89 & 85 \\
\hline 9 & 71 & 50 & 68 & 68 & 68 & 61 & 70 & 59 \\
\hline 10 & 46 & 62 & 43 & 64 & 53 & 54 & 44 & 63 \\
\hline 11 & 45 & 60 & 42 & 57 & 49 & 53 & 43 & 59 \\
\hline 12 & 86 & 86 & 92 & 79 & 85 & 86 & 89 & 82 \\
\hline
\end{tabular}

Fonte: elaborado pelos autores. 


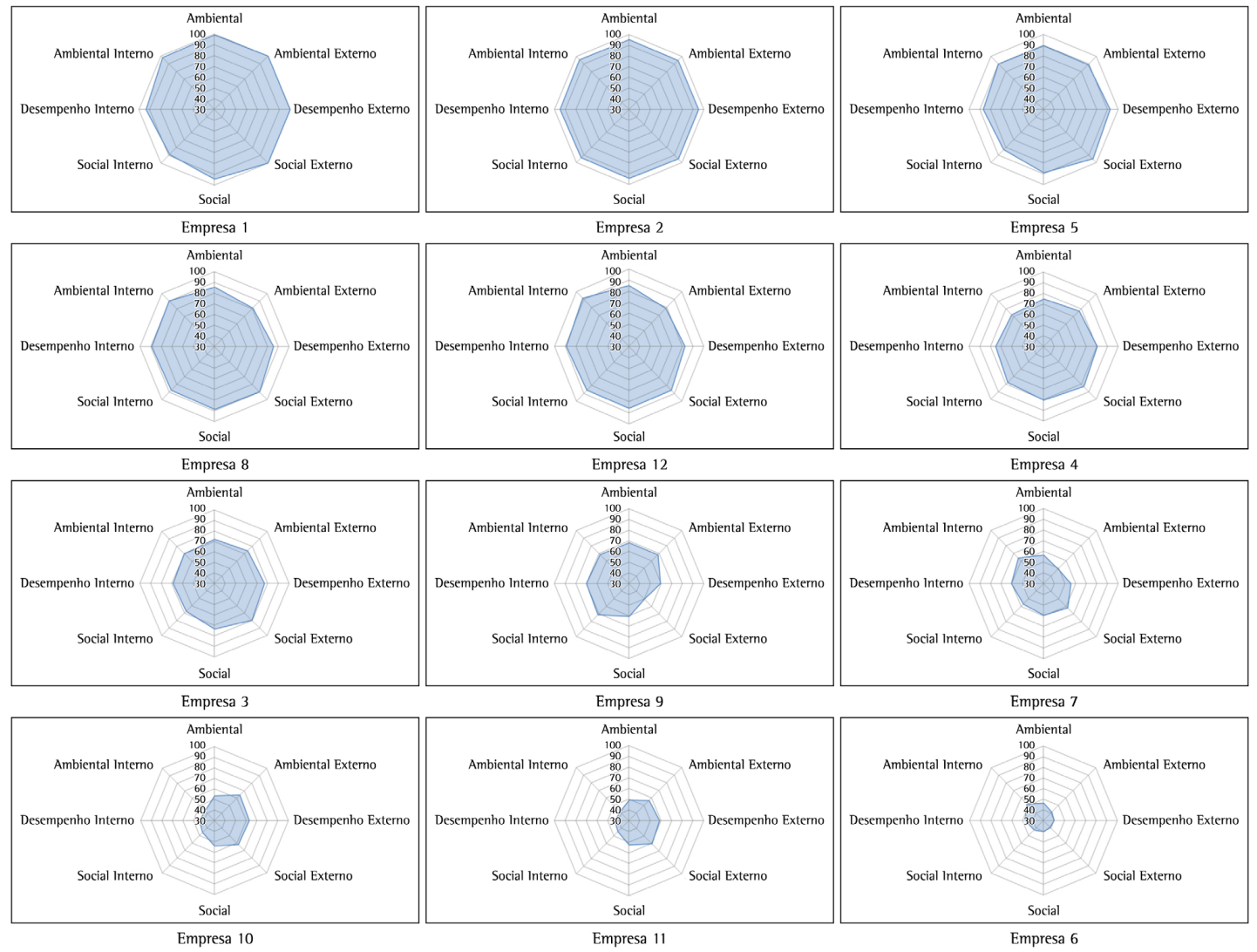

Figura 1. Avaliação da sustentabilidade das empresas estudadas. Fonte: elaborado pelos autores.

As empresas representadas pelos quatro últimos gráficos tiveram o pior desempenho global. Estas empresas têm como principal cliente o mercado interno, que se caracteriza por ser menos exigente que os mercados externos. Também se pode observar nos gráficos que existe um desequilíbrio entre as dimensões analisadas.

De forma geral, as empresas apresentam um melhor desempenho externo, o que pode ser decorrente da preocupação com a sua imagem frente à sociedade e, principalmente, aos seus clientes. No que diz respeito aos quadrantes, o melhor desempenho está no social externo, ao passo que o pior desempenho é observado para o quadrante social interno.

Observando-se as dimensões social e ambiental, as empresas apresentaram igual desempenho. Este comportamento não era esperado, uma vez que há um número superior de empresas que possuem um sistema de gestão ambiental implantado a um tempo mais longo.

0 procedimento analítico e gráfico utilizado mostrou-se de fácil aplicação, integrando as dimensões sociais e ambientais, e contemplando os aspectos internos e externos da empresa. A visualização gráfica facilita a rápida apreensão e comunicação dos resultados. Outra vantagem do método é o foco no nível organizacional, diferentemente da maioria dos métodos encontrados na literatura, que têm foco em nível de produto e abordam principalmente a dimensão ambiental (Azapagic \& Perdan, 2000; Ness et al., 2007), não indicando a sustentabilidade geral da empresa. Observa-se, como uma limitação do procedimento, o fato de a avaliação do desenvolvimento socioambiental ser realizada pela própria empresa, o que pode comprometer os resultados. Porém, considerando-se o objetivo do procedimento, que é auxiliar as empresas na gestão e no monitoramento do desenvolvimento sustentável, identificando seus pontos deficitários, esta não é uma limitação importante.

Recomenda-se que o procedimento desenvolvido seja aplicado utilizando-se amostras maiores e experimentos planejados, em que os fatores poderiam ser: porte da empresa, setor de atividade, região, entre outros. lsso permitiria comparações estatísticas do desempenho de diferentes segmentos industriais. 


\section{Conclusões}

As empresas têm dado maior importância para a sustentabilidade, entretanto esse conceito é compreendido apenas intuitivamente, sendo difícil expressá-lo em nível operacional. A avaliação da sustentabilidade representa um papel fundamental no alcance da sustentabilidade, visto que indica os pontos a ser melhorados. A partir das informações de desempenho obtidas, as empresas podem tomar decisões direcionadas à sustentabilidade. Assim, identifica-se uma intensa demanda por formas de avaliação da sustentabilidade.

0 objetivo deste trabalho foi o desenvolvimento de um procedimento analítico e gráfico para avaliação da sustentabilidade. Tal procedimento possibilita que as empresas identifiquem seu desempenho global em relação à sustentabilidade, suas dimensões (social e ambiental) e espaço de abrangência (interno e externo). 0 procedimento proposto permite a rápida apreensão e comunicação dos resultados, além de ser facilmente aplicado.

Para o desenvolvimento do procedimento, empregaram-se cinco etapas, sendo estas: identificação dos itens de avaliação; organização dos itens em dimensões a serem analisadas; aplicação do questionário; totalização dos resultados; visualização gráfica dos resultados. Para testar o procedimento desenvolvido, o questionário foi enviado a empresas de diferentes setores industriais, não sendo encontradas dificuldades em sua aplicação.

De acordo com a totalização e a visualização dos resultados, pode-se verificar que as empresas, de maneira geral, apresentam um melhor desempenho socioambiental externo, fator que pode ser oriundo da preocupação com a sua imagem frente à sociedade $\mathrm{e}$ aos seus clientes. Também se observou que empresas exportadoras possuem um melhor desempenho socioambiental do que empresas que atuam no mercado interno, o que pode ser decorrente da maior concorrência e exigência do mercado externo. Além disso, verificou-se igual desempenho nas dimensões social e ambiental, sendo este comportamento inesperado, uma vez que os dados e a literatura demonstram que as empresas preocupam-se principalmente com a dimensão ambiental.

Destaca-se que o procedimento analítico e gráfico de avaliação da sustentabilidade socioambiental empresarial desenvolvido mostrou-se uma ferramenta eficaz em seu propósito. Assim, o procedimento pode ser empregado nas empresas como forma de gestão e monitoramento de sua sustentabilidade, auxiliando para o alcance da mesma.

\section{Referências}

Análise Editorial. (2007). Anuário 2007: Gestão Ambiental. São Paulo: Análise Editorial. 386 p.

Associação Brasileira de Normas Técnicas - ABNT. (2004). NBR ISO 14001: sistemas da gestão ambiental: requisitos com orientações para uso informação e documentação: referências, elaboração. Rio de Janeiro: ABNT.

Azapagic, A. (2003). Systems approach to corporate sustainability: a general management framework. Trans IChemE, 81(5), 303-316. http://dx.doi.org/10.1205/095758203770224342.

Azapagic, A., \& Perdan, S. (2000). Indicators of sustainable development for industry: a general framework. Trans IChemE, 78(4), 243-261. http://dx.doi.org/10.1205/095758200530763.

BM\&FBOVESPA. (2008). Índice de Sustentabilidade Empresarial: questionário base. São Paulo: Bovespa. 262 p.

Böhringer, C., \& Jochem, P. E. P. (2007). Measuring the immeasurable: a survey of sustainability indices. Ecological Economics, 63(1), 1-8. http://dx.doi.org/10.1016/j. ecolecon.2007.03.008.

Bossel, H. (1999). Indicators for sustainable development: theory, method, applications: a report to the Balaton group. Winnipeg: IISD.

Brasil. Ministério do Meio Ambiente. (1992). Agenda 21 global. Brasília: MMA. Recuperado em 20 Jan. 2016, de http://www. mma.gov.br/responsabilidade-socioambiental/agenda-21/ agenda-21-global.

Brasil. Ministério do Meio Ambiente. (2007). Agenda 21 Brasileira. Brasília: MMA. Recuperado em 21 Ago. 2007, de http://www.mma.gov.br/index.php?ido=conteudo.mo nta\&idEstrutura=18\&idConteudo=908.

Brown, H. S., Jong, M., \& Levy, D. L. (2009). Building institutions based on information disclosure: lessons from GRl's sustainability reporting. Journal of Cleaner Production, 17(6), 571-580. http://dx.doi.org/10.1016/j. jclepro.2008.12.009.

Cantarino, A. A. A., Barata, M., \& Rovere, E. L. L. (2007) Indicadores de sustentabilidade empresarial e gestão estratégica. Revista pensamento contemporâneo em administração, 1, 115-130.

Darby, L., \& Jenkins, H. (2006). Applying sustainability indicators to the social enterprise business model: the development and application of an indicator set for Newport Wastesavers, Wales. International Journal of Social Economics, 33(56), 411-431.

Figge, F., \& Hahn, T. (2004). Sustainable value added: measuring corporate contributions to sustainability beyond ecoefficiency. Ecological Economics, 48(2), 173-187. http:// dx.doi.org/10.1016/j.ecolecon.2003.08.005.

Garcia-Serna, J., Perez-Barrigon, L., \& Cocero, M. J. (2007). New trends for design towards sustainability in chemical engineering: green engineering. Chemical Engineering Journal, 133(1-3), 7-30. http://dx.doi.org/10.1016/j. cej.2007.02.028.

Gasparatos, A., El-Haram, M., \& Horner, M. (2008). A critical review of reductionist approaches for assessing the progress towards sustainability. Environmental Impact Assessment Review, 28(4-5), 286-311. http://dx.doi.org/10.1016/j. eiar.2007.09.002.

Hardi, P., \& Zdan, T. J. (2000). The dashboard of sustainability (Draft paper). Winnipeg: IISD. 
Hart, S. L., \& Milstein, M. B. (2004). Criando valor sustentável. RAE executivo, 3(2), 65-79.

Herzig, C., \& Schaltegger, S. (2006). Corporate sustainability reporting: an overview. In S. SCHALTEGGER, M. BENNETT \& R. BURRITT (Ed.), Sustainability accounting and reporting (cap. 13, p. 301-324). Dordrecht: Springer.

Instituto Ethos de Empresas e Responsabilidade Social - ETHOS. (2006a). Global Reporting Initiative: diretrizes para relatório de sustentabilidade. São Paulo: ETHOS. 47 p.

Instituto Ethos de Empresas e Responsabilidade Social - ETHOS. (2006b). Instituto Ethos de Responsabilidade Social Empresarial. São Paulo: ETHOS. 80 p.

Krajnc, D., \& Glavic, P. (2003). Indicators of sustainable production. Clean Technologies and Environmental Policy, 5(3-4), 279-288. http://dx.doi.org/10.1007/s10098-003-0221-z.

Krajnc, D., \& Glavic, P. (2005). A model for integrated assessment of sustainable development. Resources, Conservation and Recycling, 43(2), 189-208. http://dx.doi.org/10.1016/ S0921-3449(04)00120-X.

Labuschagne, C., Brent, A. C., \& van Erck, R. P. G. (2005). Assessing the sustainability performances of industries. Journal of Cleaner Production, 13(4), 373-385. http:// dx.doi.org/10.1016/j.jclepro.2003.10.007.

Lozano, R. (2006). A tool for a Graphical Assessment of Sustainability in Universities (GASU). Journal of Cleaner Production, 14(9-11), 963-972. http://dx.doi.org/10.1016/j. jclepro.2005.11.041.

Ness, B., Urbel-Piirsalu, E., Anderberg, S., \& Olsson, L. (2007). Categorising tools for sustainability assessment. Ecological Economics, 60(3), 498-508. http://dx.doi.org/10.1016/j. ecolecon.2006.07.023.

Parris, T. M., \& Kates, R. W. (2003). Characterizing and measuring sustainable development. Annual Review of Environment and Resources, 28(1), 559-586. http://dx.doi.org/10.1146/ annurev.energy.28.050302.105551.

Phillis, Y. A., \& Davis, B. J. (2009). Assessment of corporate sustainability via fuzzy logic. Journal of Intelligent \& Robotic Systems, 55(1), 3-20. http://dx.doi.org/10.1007/ s10846-008-9289-3.
Prescott-Allen, R. (1997). Barometer of sustainability: measuring and communicating wellbeing and sustainable development. Cambridge: IUCN.

Programa das Nações Unidas para o Meio Ambiente - PNUMA. (2002). Integração entre o meio ambiente e o desenvolvimento: 1972-2002. In Perspectivas do Meio Ambiente Mundial GEO-03 (pp. 1-28). Brasília: PNUMA.

Revista Amanhã. (2008, Junho). Teste amanhã de sustentabilidade. Revista Amanhã, 243.

Singh, R. K., Murty, H. R., Gupta, S. K., \& Dikshit, A. K. (2009). An overview of sustainability assessment methodologies. Ecological Indicators, 9(2), 189-212. http://dx.doi. org/10.1016/j.ecolind.2008.05.011.

Ugwu, O., \& Haupt, T. (2007). Key performance indicators and assessment methods for infrastructure sustainability: south african construction industry perspective. Building and Environment, 42(2), 665-680. http://dx.doi.org/10.1016/j. buildenv.2005.10.018.

Veiga, L. B. E., \& Magrini, A. (2009). Eco-industrial park development in Rio de Janeiro, Brazil: a tool for sustainable development. Journal of Cleaner Production, 17(7), 653661. http://dx.doi.org/10.1016/j.jclepro.2008.11.009.

Veleva, V., Bailey, J., \& Jurczyk, N. (2001). Using sustainable production indicators to measure progress in ISO 14001, EHS system and EPA achievement track. Corporate Environmental Strategy, 8(4), 326-338. http://dx.doi. org/10.1016/S1066-7938(01)00138-5.

Wang, L., \& Lin, L. (2004). Making sustainability accountable: a valuation model for corporate performance. In IEEE 2004 International Symposium on Electronics and the Environment (pp. 7-12), Washington, D.C, USA.

Weber, M. (2008). The business case for corporate social responsibility: a company-level measurement approach for CSR. European Management Journal, 26(4), 247-261. http://dx.doi.org/10.1016/j.emj.2008.01.006.

Wilson, J., Tyedmers, P., \& Pelot, R. (2007). Contrasting and comparing sustainable development indicator metrics. Ecological Indicators, 7(2), 299-314. http://dx.doi. org/10.1016/j.ecolind.2006.02.009.

World Commission on Environment and Development - WCED. (1987). Our common future. Oxford: Oxford University Press. 383 p.

\title{
A proposal for social and environmental sustainability evaluation using analytical and graphical support
}

\begin{abstract}
This paper presents a procedure to assist companies conducting socioenvironmental sustainability evaluations, an aspect that has become essential for competitiveness. The proposed procedure applies analytical and graphical tools to evaluate companies' social and environmental dimensions, considering internal and external performance. The development of the proposed procedure comprised five steps: identification of evaluation items, organization of items in dimensions to be analyzed, application of questionnaire, summation, and graphical visualization of results. The questionnaire was applied to 12 companies. A radar graphic was employed for results visualization, where vertical axes represent social and environmental dimensions while horizontal axes represent internal and external dimensions. Surveyed companies revealed higher social and environmental performance in the external dimension, probably due to concern with their market image. It was also observed that exporting companies presented superior social and environmental performance, fact that might be attributed to the more rigorous demand of external market.
\end{abstract}

\section{Keywords}

Sustainability evaluation. Social and environmental sustainability. Social responsibility. Environmental management. 
Teles, C. D. et al.

Uma proposta para avaliação da sustentabilidade ... suporte analítico e gráfico. Production, 26(2), 417-429, abr./jun. 2016

Apêndice A. Itens de avaliação e dimensões avaliadas.

\begin{tabular}{|c|c|c|c|c|c|}
\hline & ltens & SI & SE & $\mathrm{AE}$ & $\mathrm{Al}$ \\
\hline 1 & $\begin{array}{l}\text { A organização possui um código de ética/conduta, ou documento equivalente, que expressa os seus valores } \\
\text { e princípios? }\end{array}$ & $x$ & $x$ & $x$ & $\mathrm{x}$ \\
\hline 2 & 0 código de ética é amplamente divulgado em todos os níveis hierárquicos? & $\mathrm{x}$ & $\mathrm{x}$ & $\mathrm{x}$ & $\mathrm{x}$ \\
\hline 3 & $\begin{array}{l}0 \text { código de ética ou declaração de princípios proíbe expressamente práticas ilegais (como concorrência } \\
\text { desleal, pirataria, propina e “caixa dois”) para a obtenção de vantagens nos negócios? }\end{array}$ & $x$ & $x$ & $x$ & $x$ \\
\hline 4 & A empresa tem conselhos, comitês ou grupos responsáveis pelas questões éticas? & $\mathrm{x}$ & $\mathrm{x}$ & $\mathrm{x}$ & $\mathrm{x}$ \\
\hline 5 & $\begin{array}{l}\text { Os princípios do Desenvolvimento Sustentável, considerados os aspectos social e ambiental, estão } \\
\text { incorporados ao planejamento estratégico? }\end{array}$ & $x$ & & & $x$ \\
\hline 6 & Publica informações sobre sua gestão social? & $x$ & & & \\
\hline 7 & Publica informações sobre sua gestão ambiental? & & & & $\mathrm{x}$ \\
\hline 8 & $\begin{array}{l}\text { Onde as informações relativas à gestão socioambiental são publicadas? } \\
\text { ( ) Relatório anual ( ) BS/Modelo GRl } \\
\text { ( ) 1AN (Informações Anuais) ( ) BS/Modelo IBASE } \\
\text { ( ) Balanço socioambiental ( ) BS/Modelo Ethos } \\
\text { ( ) Site da empresa ( ) Outro. Qual? } \\
\text { ( ) BS (Balanço Social) }\end{array}$ & $x$ & & & $x$ \\
\hline $9 a$ & $\begin{array}{l}\text { A comunicação das ações sociais é voltada a: } \\
\text { ( ) Escola ( ) Público em geral ( ) Vizinhança } \\
\text { ( ) ONGs ( ) Autoridades ( ) Outros. Quais? } \\
\text { ( ) Não há programa }\end{array}$ & $\mathrm{x}$ & & & \\
\hline $9 \mathrm{~b}$ & $\begin{array}{l}\text { A comunicação das ações ambientais é voltada a: } \\
\text { ( ) Escola ( ) Público em geral ( ) Vizinhança } \\
\text { ( ) ONGs ( ) Autoridades ( ) Outros. Quais? } \\
\text { ( ) Não há programa }\end{array}$ & & & & $x$ \\
\hline 10 & $\begin{array}{l}\text { Possui 1SO } 9001 \text { ou norma equivalente? } \\
\text { ( ) Sim, há até } 2 \text { anos ( ) Sim, mais de } 10 \text { anos } \\
\text { ( ) Sim, de } 2 \text { a } 5 \text { anos ( ) Não, mas planeja implementar } \\
\text { ( ) Sim, de } 5 \text { a } 10 \text { anos ( ) Não }\end{array}$ & & & & $\mathrm{x}$ \\
\hline 11 & $\begin{array}{l}\text { Possui ISO } 14001 \text { ou norma equivalente? } \\
\text { ( ) Sim, há até } 2 \text { anos ( ) Sim, mais de } 10 \text { anos } \\
\text { ( ) Sim, de } 2 \text { a } 5 \text { anos ( ) Não, mas planeja implementar } \\
\text { ( ) Sim, de } 5 \text { a } 10 \text { anos ( ) Não }\end{array}$ & $x$ & $x$ & $x$ & $x$ \\
\hline 12 & $\begin{array}{l}\text { Possui OHSAS } 18001 \text { ou norma equivalente? } \\
\text { ( ) Sim, há até } 2 \text { anos ( ) Sim, mais de } 10 \text { anos } \\
\text { ( ) Sim, de } 2 \text { a } 5 \text { anos ( ) Não, mas planeja implementar } \\
\text { ( ) Sim, de } 5 \text { a } 10 \text { anos ( ) Não }\end{array}$ & & & & $x$ \\
\hline 13 & Possui política ambiental? & $\mathrm{x}$ & & & \\
\hline 14 & A responsabilidade pela gestão ambiental está definida? & & & & $\mathrm{x}$ \\
\hline 15 & Possui política de responsabilidade social? & & & & $\mathrm{x}$ \\
\hline 16 & A responsabilidade pela gestão da responsabilidade social está definida? & $\mathrm{x}$ & & & \\
\hline 17 & $\begin{array}{l}\text { Nos procedimentos adotados relacionados à gestão ambiental, a empresa se preocupa em: } \\
\text { ( ) cumprir rigorosamente os parâmetros legais. } \\
\text { ( ) desenvolver ações para prevenir os impactos ambientais causados por seus processos e produtos ou } \\
\text { serviços. } \\
\text { ( ) realizar regularmente programas de melhorias e atividades de controle e monitoramento. }\end{array}$ & $x$ & & & \\
\hline 18 & $\begin{array}{l}\text { A empresa: } \\
\text { ( ) produz estudos de impacto em toda a cadeia produtiva. } \\
\text { ( ) desenvolve parcerias com os fornecedores para melhorar seus processos e minimizar os impactos. } \\
\text { ( ) desenvolve parcerias com outras instituições para melhorar seus processos e minimizar os impactos. }\end{array}$ & & & & $\mathrm{x}$ \\
\hline 19 & $\begin{array}{l}\text { Possui normas conhecidas de seleção e avaliação de fornecedores que contemplam exigências e critérios } \\
\text { relativos ao cumprimento da legislação trabalhista, previdenciária, fiscal e ambiental? }\end{array}$ & & & $x$ & \\
\hline 20 & $\begin{array}{l}\text { Fornece aos clientes e consumidores informações detalhadas sobre danos ambientais resultantes do uso e } \\
\text { da destinação final de seus produtos? }\end{array}$ & & $x$ & $x$ & \\
\hline 21 & $\begin{array}{l}\text { Conhece profundamente a origem dos insumos, matérias-primas e produtos utilizados em suas operações, e } \\
\text { tem a garantia de que, nessas origens, os direitos humanos e o meio ambiente são respeitados? }\end{array}$ & & & $x$ & \\
\hline 22 & $\begin{array}{l}\text { Possui política de compras que privilegie aqueles fornecedores que possuem certificações socioambientais } \\
\text { (como SA8000, ISO 14000, FSC, FLO etc)? }\end{array}$ & & $\mathrm{x}$ & $x$ & \\
\hline 23 & $\begin{array}{l}\text { Possui procedimentos formais para recebimento, registro e resposta às demandas das partes interessadas } \\
\text { (stakeholders)? }\end{array}$ & & $\mathrm{x}$ & $x$ & \\
\hline 24 & $\begin{array}{l}\text { Tais impactos ambientais referem-se a: } \\
\text { ( ) processos produtivos. } \\
\text { ( ) administrativos. } \\
\text { ( ) terceirizados. } \\
\text { ( ) fornecedores. }\end{array}$ & & & $x$ & $x$ \\
\hline
\end{tabular}

Fonte: elaborado pelos autores. 
Apêndice A. Continuação...

\begin{tabular}{|c|c|c|c|c|c|}
\hline & Itens & $\mathrm{SI}$ & SE & $\mathrm{AE}$ & $\mathrm{Al}$ \\
\hline 25 & Monitora com indicadores os resíduos (sólidos, efluentes, emissões atmosféricas, ruídos e vibrações)? & & & & $\mathrm{x}$ \\
\hline 26 & Tem metas de redução, reuso e reciclagem dos resíduos? & & & & $\mathrm{x}$ \\
\hline 27 & Investe em tecnologia para redução da geração de resíduos? & & & & $\mathrm{x}$ \\
\hline 28 & Faz coleta seletiva do lixo? & & & & $\mathrm{x}$ \\
\hline 29 & $\begin{array}{l}\text { Monitora com indicadores os recursos naturais (água, energia elétrica, combustíveis, lenha e carvão, e } \\
\text { recursos minerais)? }\end{array}$ & & & & $\mathrm{x}$ \\
\hline 30 & Tem metas de redução do uso de recursos naturais? & & & & $\mathrm{x}$ \\
\hline 31 & $\begin{array}{l}\text { Possui sistema de gerenciamento e coleta de materiais tóxicos ou potencialmente danosos ao ambiente e à } \\
\text { saúde? }\end{array}$ & & & & $\mathrm{x}$ \\
\hline 32 & Possui projetos que contribuem para o desenvolvimento dos filhos dos empregados? & $\mathrm{x}$ & & & \\
\hline 33 & Apoia projetos focados nas crianças e adolescentes da comunidade? & & $\mathrm{x}$ & & \\
\hline 34 & $\begin{array}{l}\text { Cumpre rigorosamente as obrigações legais e tem metas para alcançar padrões de excelência em saúde, } \\
\text { segurança e condições de trabalho (inclusive com indicadores para monitorar o alcance dessas metas)? }\end{array}$ & $\mathrm{x}$ & & & \\
\hline 35 & $\begin{array}{l}\text { Tem políticas de desenvolvimento e capacitação, visando ao aperfeiçoamento contínuo de todo o seu } \\
\text { pessoal? }\end{array}$ & $\mathrm{x}$ & & & \\
\hline 36 & $\begin{array}{l}\text { Além de promover a capacitação, oferece bolsas de estudos ou similares, de forma a melhorar a } \\
\text { empregabilidade dos funcionários? }\end{array}$ & $\mathrm{x}$ & & & \\
\hline 37 & $\begin{array}{l}\text { Mantém programa de erradicação do analfabetismo (absoluto e/ou funcional), de ensino supletivo ou de } \\
\text { conclusão da educação básica pelos empregados, com metas e recursos definidos? }\end{array}$ & $\mathrm{x}$ & & & \\
\hline 38 & $\begin{array}{l}\text { Em relação à diversidade, há políticas explícitas de não discriminação (raça, gênero, idade, religião e } \\
\text { orientação sexual) na política salarial, na admissão, na promoção, no treinamento e na demissão de } \\
\text { empregados? }\end{array}$ & $\mathrm{x}$ & & & \\
\hline 39 & $\begin{array}{l}\text { Cumpre a legislação sobre a contratação de pessoas com deficiência e mantém programas para a inserção } \\
\text { dessas pessoas na empresa? }\end{array}$ & $\mathrm{x}$ & & & \\
\hline 40 & Possui Plano de Participação nos Resultados para os empregados? & $\mathrm{x}$ & & & \\
\hline 41 & $\begin{array}{l}\text { Possui políticas formais com o objetivo de contribuir para a erradicação do trabalho infantil e do trabalho } \\
\text { forçado, discutindo a questão com os fornecedores e estimulando-os a cumprir a lei? }\end{array}$ & & $\mathrm{x}$ & & \\
\hline 42 & $\begin{array}{l}\text { Apoia - técnica ou financeiramente - políticas públicas e ações (governamentais ou de entidades do } \\
\text { terceiro setor) focadas na melhoria da saúde pública e da educação, na erradicação do trabalho infantil e } \\
\text { em outras ações de fortalecimento da cidadania? }\end{array}$ & & $\mathrm{x}$ & & \\
\hline
\end{tabular}

Apêndice B. Compilação das respostas das empresas.

\begin{tabular}{|c|c|c|c|c|c|c|c|c|c|c|c|c|}
\hline \multirow{2}{*}{ Itens } & \multicolumn{12}{|c|}{ Empresas } \\
\hline & 1 & 2 & 3 & 4 & 5 & 6 & 7 & 8 & 9 & 10 & 11 & 12 \\
\hline 1 & 10,00 & 10,00 & 10,00 & 10,00 & 10,00 & 8,00 & 10,00 & 10,00 & 10,00 & 10,00 & 9,00 & 10,00 \\
\hline 2 & 10,00 & 10,00 & 10,00 & 10,00 & 10,00 & 6,00 & 6,00 & 10,00 & 10,00 & 7,00 & 8,00 & 10,00 \\
\hline 3 & 10,00 & 10,00 & 9,00 & 10,00 & 10,00 & 8,00 & 8,00 & 10,00 & 8,00 & 10,00 & 0,00 & 10,00 \\
\hline 4 & 10,00 & 10,00 & 8,00 & 10,00 & 10,00 & 9,00 & 5,00 & 10,00 & 10,00 & 0,00 & 0,00 & 10,00 \\
\hline 5 & 10,00 & 8,00 & 8,00 & 10,00 & 10,00 & 6,00 & 9,00 & 8,00 & 9,00 & 9,00 & 8,00 & 10,00 \\
\hline 6 & 10,00 & 8,00 & 9,00 & 9,00 & 10,00 & 5,00 & 6,00 & 9,00 & 9,00 & 2,00 & 0,00 & 10,00 \\
\hline 7 & 10,00 & 8,00 & 9,00 & 9,00 & 10,00 & 0,00 & 2,00 & 9,00 & 9,00 & 2,00 & 0,00 & 10,00 \\
\hline 8 & 3,75 & 2,50 & 3,75 & 1,25 & 2,50 & 1,25 & 9,00 & 2,50 & 2,50 & 1,25 & 1,25 & 3,33 \\
\hline 9a & 10,00 & 10,00 & 0,00 & 2,00 & 8,00 & 2,00 & 1,00 & 4,00 & 4,00 & 2,00 & 4,00 & 7,33 \\
\hline $9 b$ & 10,00 & 10,00 & 0,00 & 2,00 & 10,00 & 2,00 & 5,00 & 4,00 & 2,00 & 2,00 & 4,00 & 8,00 \\
\hline 10 & 10,00 & 10,00 & 8,00 & 4,00 & 2,00 & 6,00 & 4,00 & 10,00 & 6,00 & 2,00 & 6,00 & 10,00 \\
\hline 11 & 10,00 & 10,00 & 2,00 & 2,00 & 2,00 & 0,00 & 6,00 & 10,00 & 2,00 & 0,00 & 6,00 & 8,00 \\
\hline 12 & 8,00 & 8,00 & 2,00 & 0,00 & 0,00 & 2,00 & 0,00 & 8,00 & 2,00 & 0,00 & 6,00 & 1,33 \\
\hline 13 & 10,00 & 10,00 & 9,00 & 10,00 & 10,00 & 7,00 & 0,00 & 10,00 & 6,00 & 10,00 & 10,00 & 10,00 \\
\hline 14 & 10,00 & 10,00 & 8,00 & 10,00 & 10,00 & 7,00 & 5,00 & 10,00 & 6,00 & 10,00 & 9,00 & 9,67 \\
\hline 15 & 4,00 & 10,00 & 8,00 & 10,00 & 10,00 & 6,00 & 9,00 & 10,00 & 6,00 & 6,00 & 10,00 & 9,67 \\
\hline 16 & 10,00 & 10,00 & 7,00 & 8,00 & 10,00 & 5,00 & 7,00 & 10,00 & 6,00 & 6,00 & 9,00 & 9,33 \\
\hline 17 & 10,00 & 10,00 & 10,00 & 3,33 & 10,00 & 3,33 & 5,00 & 10,00 & 10,00 & 3,33 & 10,00 & 8,89 \\
\hline 18 & 10,00 & 10,00 & 3,33 & 3,33 & 6,66 & 3,33 & 0,00 & 6,66 & 6,66 & 3,33 & 3,33 & 8,89 \\
\hline 19 & 10,00 & 8,00 & 8,00 & 10,00 & 10,00 & 1,00 & 8,00 & 8,00 & 10,00 & 8,00 & 9,00 & 9,33 \\
\hline 20 & 10,00 & 9,00 & 9,00 & 8,00 & 10,00 & 1,00 & 4,00 & 9,00 & 2,00 & 9,00 & 6,00 & 4,33 \\
\hline
\end{tabular}

Fonte: elaborado pelos autores. 
Teles, C. D. et al.

Uma proposta para avaliação da sustentabilidade ... suporte analítico e gráfico. Production, 26(2), 417-429, abr./jun. 2016

Apêndice B. Continuação...

\begin{tabular}{|c|c|c|c|c|c|c|c|c|c|c|c|c|}
\hline \multirow{2}{*}{ Itens } & \multicolumn{12}{|c|}{ Empresas } \\
\hline & 1 & 2 & 3 & 4 & 5 & 6 & 7 & 8 & 9 & 10 & 11 & 12 \\
\hline 21 & 10,00 & 10,00 & 9,00 & 8,00 & 9,00 & 2,00 & 6,00 & 9,00 & 6,00 & 8,00 & 7,00 & 7,00 \\
\hline 22 & 10,00 & 8,00 & 8,00 & 7,00 & 9,00 & 2,00 & 6,00 & 7,00 & 3,00 & 6,00 & 8,00 & 4,33 \\
\hline 23 & 10,00 & 10,00 & 8,00 & 9,00 & 10,00 & 5,00 & 7,00 & 5,00 & 10,00 & 5,00 & 6,00 & 10,00 \\
\hline 24 & 10,00 & 10,00 & 5,00 & 10,00 & 10,00 & 10,00 & 2,50 & 7,50 & 10,00 & 7,50 & 5,00 & 8,33 \\
\hline 25 & 10,00 & 10,00 & 7,00 & 9,00 & 10,00 & 6,00 & 8,00 & 10,00 & 6,00 & 7,00 & 3,00 & 9,67 \\
\hline 26 & 10,00 & 10,00 & 6,00 & 9,00 & 10,00 & 8,00 & 8,00 & 10,00 & 8,00 & 7,00 & 1,00 & 9,33 \\
\hline 27 & 10,00 & 10,00 & 8,00 & 9,00 & 10,00 & 5,00 & 8,00 & 8,00 & 1,00 & 2,00 & 2,00 & 8,67 \\
\hline 28 & 10,00 & 10,00 & 9,00 & 10,00 & 10,00 & 9,00 & 10,00 & 10,00 & 9,00 & 2,00 & 1,00 & 10,00 \\
\hline 29 & 10,00 & 10,00 & 8,00 & 5,00 & 9,00 & 6,00 & 9,00 & 10,00 & 9,00 & 2,00 & 0,00 & 10,00 \\
\hline 30 & 10,00 & 10,00 & 7,00 & 5,00 & 9,00 & 2,00 & 9,00 & 10,00 & 9,00 & 2,00 & 0,00 & 8,67 \\
\hline 31 & 10,00 & 10,00 & 8,00 & 9,00 & 10,00 & 9,00 & 8,00 & 10,00 & 9,00 & 2,00 & 7,00 & 10,00 \\
\hline 32 & 4,00 & 10,00 & 8,00 & 5,00 & 8,00 & 1,00 & 7,00 & 7,00 & 10,00 & 2,00 & 0,00 & 8,67 \\
\hline 33 & 10,00 & 10,00 & 8,00 & 9,00 & 10,00 & 5,00 & 7,00 & 10,00 & 6,00 & 2,00 & 9,00 & 10,00 \\
\hline 34 & 10,00 & 10,00 & 8,00 & 10,00 & 10,00 & 4,00 & 8,00 & 10,00 & 8,00 & 8,00 & 6,00 & 10,00 \\
\hline 35 & 10,00 & 10,00 & 8,00 & 9,00 & 10,00 & 6,00 & 8,00 & 9,00 & 8,00 & 7,00 & 8,00 & 10,00 \\
\hline 36 & 10,00 & 10,00 & 7,00 & 10,00 & 8,00 & 0,00 & 6,00 & 9,00 & 2,00 & 4,00 & 2,00 & 9,67 \\
\hline 37 & 10,00 & 9,00 & 6,00 & 9,00 & 10,00 & 0,00 & 0,00 & 10,00 & 8,00 & 2,00 & 2,00 & 6,67 \\
\hline 38 & 10,00 & 10,00 & 8,00 & 10,00 & 10,00 & 5,00 & 7,00 & 10,00 & 9,00 & 9,00 & 9,00 & 9,67 \\
\hline 39 & 10,00 & 10,00 & 8,00 & 10,00 & 10,00 & 9,00 & 8,00 & 10,00 & 10,00 & 5,00 & 2,00 & 9,67 \\
\hline 40 & 10,00 & 10,00 & 7,00 & 10,00 & 6,00 & 7,00 & 7,00 & 10,00 & 10,00 & 5,00 & 0,00 & 10,00 \\
\hline 41 & 10,00 & 9,00 & 7,00 & 5,00 & 10,00 & 5,00 & 6,00 & 9,00 & 0,00 & 9,00 & 7,00 & 6,67 \\
\hline 42 & 10,00 & 10,00 & 7,00 & 10,00 & 10,00 & 0,00 & 6,00 & 10,00 & 0,00 & 7,00 & 1,00 & 9,33 \\
\hline
\end{tabular}

